\title{
Akt: a new activation mechanism
}

\author{
Cell Research (2014) 24:785-786. doi:10.1038/cr.2014.57; published online 6 May 2014
}

\begin{abstract}
Akt phosphorylation at $\mathrm{S} 473$ and T308 has been believed to be the prerequisites for its activation for years. Now, new phosphorylation event on Akt is identified and can trigger Akt activation and lead to its downstream oncogenic events.
\end{abstract}

Protein Kinase B (PKB), more commonly referred to as Akt, is a serine/ threonine-specific protein kinase. It is well established as a critical signaling node that regulates a myriad of cellular functions including proliferation, survival, apoptosis and metabolism, which are also processes important for tumorigenesis [1]. Indeed, the roles of Akt in cancer have been extensively scrutinized over the years and documented in many studies. However, more studies are still emerging to further increase our knowledge of this complex master regulator. In a recent paper published in Nature, Liu et al. [2] have identified a new phosphorylation event on the Akt extreme carboxyl (C)-terminus, specifically on residues S477 and T479, which activates Akt and subsequent downstream oncogenic cellular functions.

Akt activation is governed by a dual regulatory mechanism in which it is first recruited to the cellular plasma membrane by PIP3 through a direct interaction with the $\mathrm{PH}$ domain of Akt. Once Akt arrives at the plasma membrane, it meets two of its kinases PDK1 and $\mathrm{mTORC} 2$ to get phosphorylated on T308 and S473, respectively, for its full activation $[3,4]$. One way that Akt affects cell proliferation is through regulating cell cycle progression. Activated form of Akt can modulate the functions of many substrates responsible for cell-cycle progression. For example,
Akt can inactivate cell cycle inhibitors $\mathrm{p} 27^{\mathrm{kip} 1}$ and $\mathrm{p} 21^{\text {cip } 1}$ to allow G1/S progression [5]. However, it is unclear whether dysregulated cell cycle is one of the culprits for Akt hyperactivation. Liu and colleagues made great strides and uncovered a new mechanism for Akt activation linking two well-known cancer hallmarks together [6]. Liu et al. demonstrated that Akt activation fluctuates across cell cycle, which resembles the expression pattern of cyclin A2, the binding partner and activator for cyclin-dependent kinase 2 (CDK2). This prompted them to further investigate the relationship between Akt and cyclin A2. Indeed, depletion of cyclin A2, but not cyclin E, negatively affects Akt phosphorylation and activation, which is independent of changes in cell cycle and known Akt kinases PDK1 and mTORC2, suggesting that Akt is a direct substrate of cyclin A2/CDK2.

By using in vitro kinase assay and mass spectrometry, they further demonstrated that Akt can be phosphorylated by cyclin $\mathrm{A} 2 / \mathrm{CDK} 2$ within its extreme C-terminus on residues $\mathrm{S} 477$ and T479. Interestingly, this new phosphorylation event can be triggered by different upstream stimuli and different kinases. In addition to having cyclin A2/CDK2 as its kinase during cell cycle progression, S477 and T479 phosphorylation (pS477/T479) can also be mediated by mTORC2 and DNAPK under growth factor stimulation and DNA damaging conditions, respectively, as shown by their in vitro kinase assays. To address the relevancy of this modification event, they generated phospho-deficient and phospho-mimetic mutants of Akt S477/ T479. By using these mutants, Liu and colleagues concluded that pS477/T479 activates Akt and governs pS473 and pT308, which are well-characterized and widely accepted phosphorylation sites essential for Akt activation. Through structural study of Akt, they suggested the possibility that pS477/ T479 stabilizes Akt active conformation and primes pS473 by increasing its interaction with the mTORC2 complex. More importantly, phospho-mimetic mutant exhibits accelerated growth in in vitro soft agar assay while phosphodeficient mutant displays growth suppression. This result underscores the role of pS477/T479 in dictating Akt oncogenic functions.

Consistent with their in vitro observations, Liu et al. also highlighted the impact of pS477/T479 on in vivo tumor formation. Xenograft tumor model shows that phospho-mimetic mutant drives tumor growth while phosphodeficient mutant inhibits it. Remarkably, not only the new phosphorylation event positively correlates with $\mathrm{pS} 473$ in breast cancer patient samples, but also pS477/T479 can serve as a biomarker for early-stage breast cancer detection since it is frequently found in high levels in stage II breast cancer patients. This finding is considered clinically significant and can be very useful in the future for developing breast cancer prevention approaches.

This study presents compelling evidence to reveal a new mechanism for Akt activation and how Akt exerts its oncogenic functions (Figure 1). Nevertheless, there are still questions that remained to be addressed. First, since cyclin A2 is not solely localized in the cellular membrane, then where does 
Akt achieve this new phosphorylation event? Akt is known to be recruited to the cellular plasma membrane by PIP3 to be further activated by its kinases PDK1 and mTORC2. Since Liu and colleagues showed that $\mathrm{pS} 477 / \mathrm{T} 479$ not only governs $\mathrm{pS} 473$, but also pT308, the substrate site of PDK1, then one must wonder how the new phosphorylation event affects pT308. Does it affect Akt translocation to be phosphorylated by PDK1 at the plasma membrane? Another possibility is that pS477/T479 happens after Akt membrane recruitment, and simply increases the binding affinity between Akt and PDK1, similar to its effect on pS473 and mTORC2. Finally, ubiquitination of Akt on the PH domain by E3 ligases upon growth factor stimulations translocates Akt to the plasma membrane for activation and downstream biological functions such as glycolysis and tumorigenesis [7, 8]. It will also be interesting to see the crosstalk between these two important Akt modification events. The answers to these questions will certainly allow us to gain a more complete perspective on the modes of Akt activation.

Akt, being the central convergence node, participates in a wide range of influential cellular signaling pathways. Because of its pivotal role in cancer development, how it gets activated has been a prevalent area of study. Liu and colleagues have provided convincing evidence in their study that $\mathrm{pS} 477 / \mathrm{T} 479$ is a new phosphorylation event to trigger Akt activation and is important for its potency in driving tumorigenesis. Without a doubt, this article is an essential stepping-stone for us to fully understand $\mathrm{Akt}$, this intricate master regulator.

Yuan $\mathrm{Gao}^{1,2}$, Asad Moten ${ }^{1,5}$,

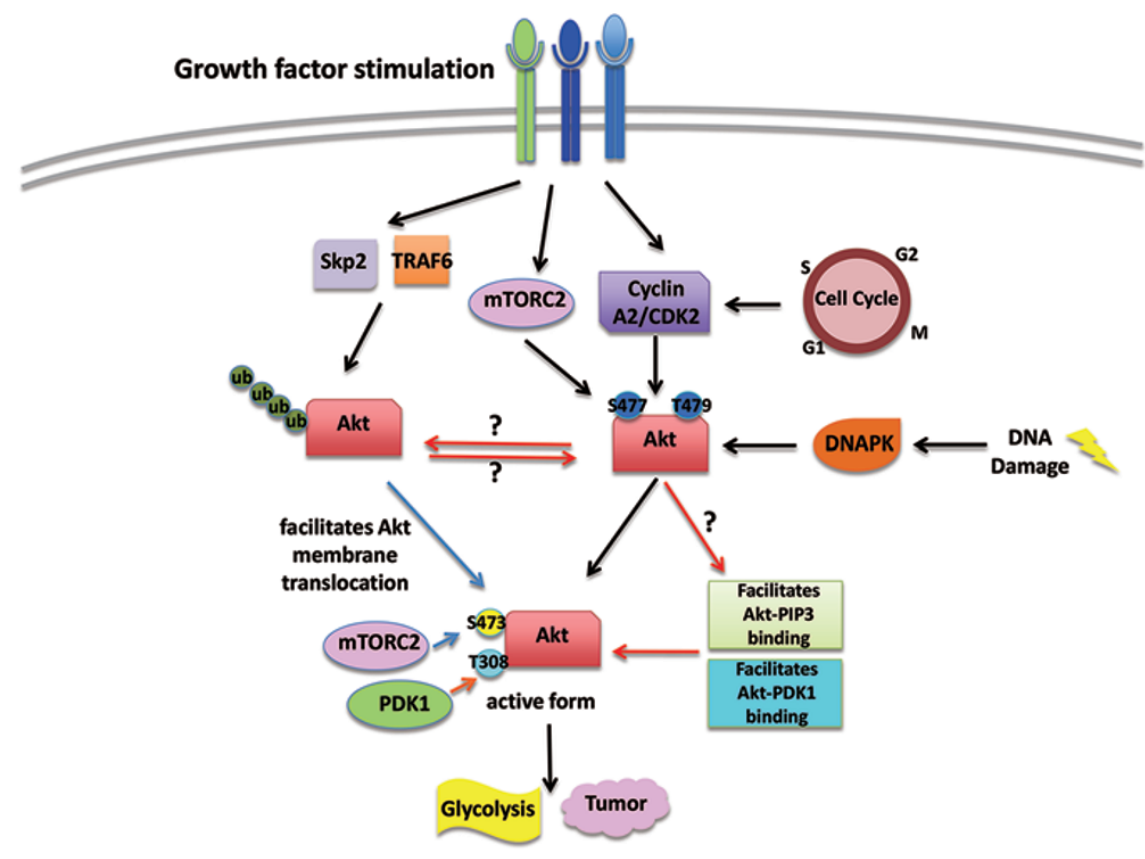

Figure 1 Akt phosphorylation triggers its activation. Liu et al. report that during cell cycle progression, under growth factor stimulation and DNA damage, Akt can be phosphorylated by cyclin A2/CDK2, mTORC2 and DNAPK. This new phosphorylation governs pS473 and pT308 to reach Akt activation. To fully understand how this modification event triggers Akt activation, we should further pursue studies that can address whether it affects Akt membrane translocation, Akt ubiquitination as well as through what mechanism it affects pT308.

\section{Hui-Kuan Lin ${ }^{1,2,3,4}$}

${ }^{1}$ Department of Molecular and Cellular Oncology, The University of Texas MD Anderson Cancer Center, Houston, TX 77030, USA; ${ }^{2}$ The University of Texas Graduate School of Biomedical Sciences at Houston, Houston, TX 77030, USA; ${ }^{3}$ Graduate Institute of Basic Medical Science, China Medical University, Taichung 404; ${ }^{4}$ Department of Biotechnology, Asia University, Taichung 404; ${ }^{5}$ Department of Primary Care Health Sciences, Nuffield Department of Clinical Medicine, University of Oxford, Oxford OX3 9DU, UK

Correspondence: Hui-Kuan Lin

E-mail: hklin@mdanderson.org

\section{References}

1 Manning BD, Cantley LC. Cell 2007; 129:1261-1274

2 Liu P, Begley M, Michowski W, et al. $\mathrm{Na}$ - ture 2014; 508:541-545.

3 Vivanco I, Sawyers CL. Nat Rev Cancer 2002; 2:489-501.

4 Sarbassov DD, Guertin DA, Ali SM, et al. Science 2005; 307:1098-1101.

$5 \quad$ Xu N, Lao Y, Zhang Y, et al. J Oncol 2012; 2012:951724.

6 Hanahan D, Weinberg RA. Cell 2011; 144:646-674.

7 Chan CH, Li CF, Yang WL, et al. Cell 2012; 149:1098-1111.

8 Yang WL, Wang J, Chan CH, et al. Science 2009; 325:1134-1138. 